\title{
现代教育技术构建氧化还原反应线上实验教学的策略探索与实践
}

\author{
魏灵灵 ${ }^{1}$ ，徐玲 ${ }^{1}$, 魏恒伟 2 , 张永策 ${ }^{3}$, 胡涛 ${ }^{3}$, 崔沝 ${ }^{3}$, 焦桓 1 , ${ }^{*}$ \\ 1 陕西师范大学化学化工学院, 西安 710119 \\ 2 陕西师范大学基础实验教学中心, 西安 710119 \\ 3 大连理工大学化工学院, 辽宁大连 116024
}

摘要: 在 “互联网 + ” 时代下, 将多种现代教育技术与翻转课堂理念相结合, 借助中国大学 MOOC 国家精品课程以 及 Mlabs 虚拟实验软件, 通过腾讯课堂构建多元化、高效化的无机化学基础实验线上课堂。以氧化还原反应线上实 验教学为例, 设计多环节教学过程、实行多方位考核评价, 在加强学生综合能力和创新能力培养的同时, 也促进教 师更新教学模式，创设符合时代发展前沿的实验内容，从而在实验教学改革的探索中实现师生共学相长。

关键词: 无机化学实验; 线上教学; MOOC; 腾讯会议; BALCKBOARD; Mlabs 虚拟仿真 中图分类号: G64; O6

\section{Exploration and Practice of Modern Educational Technology Applied in the Construction of Redox Reaction Online Laboratory Teaching}

\author{
Lingling Wei ${ }^{1}$, Ling Xu ${ }^{1}$, Hengwei Wei ${ }^{2}$, Yongce Zhang ${ }^{3}$, Tao Hu ${ }^{3}$, Miao Cui ${ }^{3}$, Huan Jiao ${ }^{1, *}$ \\ ${ }^{1}$ School of Chemistry and Chemical Engineering, Shaanxi Normal University, Xi'an 710119, P. R. China. \\ ${ }^{2}$ Basic Experimental Teaching center, Shaanxi Normal University, Xi'an 710119, P. R. China. \\ ${ }^{3}$ College of Chemistry, Dalian University of Technology, Dalian 116024, Liaoning Province, P. R. China.
}

\begin{abstract}
In the era of "Internet +", with the help of national excellent MOOC course of Chinese university and Mlabsvirtual experimental software, Tencent classroom is built to provide a diversified and efficient online inorganic chemistry basic laboratory classroom by combining a variety of modern education technologies with the concept of flipped classroom. Taking the redox reaction experiment as an example, we designed a multi-link teaching process and carried out a multi-faceted assessment. The online teaching can not only strengthen the cultivation of students' comprehensive and innovating ability, but also help teachers to update the teaching mode and create the new experimental content in line with the development of the times, so as to realize the mutual learning between teachers and students in the exploration of experimental teaching reform.
\end{abstract}

Key Words: Inorganic chemistry laboratory; Online teaching; MOOC; Tencent conference; BALCKBOARD; Mlabs-virtual simulation

\section{1 引言}

随着移动互联网、云计算、大数据、人工智能等高新科技的快速发展，“互联网+”已势如破竹 般渗透到社会生活的各个领域 ${ }^{[1]}$ 。随着教育信息化改革的持续推进, 各种线上平台迅猛发展 ${ }^{[2-4]}$ 。如 何在 “互联网+”的时代背景下对实际教学过程进行改革和更新, 从而提高学生学习兴趣、提升学习

收稿: 2020-04-09; 录用: 2020-05-12; 网络发表: 2020-05-18

“通讯作者, Email: jiaohuan@snnu.edu.cn

基金资助：陕西师范大学 2020 年度无机化学实验校级 “课程思政” 示范课建设项目 
能力、增强学习效果, 已成为理论课和实验课教学面临的重大挑战 ${ }^{[5-7]}$ 。

作为高等教育体系的重要环节, 实验课教学对于培养学生创新思维、锻炼学生实践能力、提高 学生科学素养以及拓展学生知识储备具有不可估量的重要作用。在目前疫情突发状况下, 为了全面 落实 “停课不停教、停课不停学”, 各高校针对理论课程均已开展了一个多月的线上教学实践, 而实 验课教学大多处于停滞状态 ${ }^{[8-10]}$ 。其原因在于, 相比线下真实实验操作过程, 线上教学中学生缺乏 实验过程的切身体验, 从而在很大程度上限制了其主观能动性的发挥和创造性的培养 ${ }^{[11,12]}$ 。我们基 于中国大学 MOOC 在线课程平台中的国家级精品在线开放课程一大连理工大学无机化学实验, 综 合运用滕讯会议、BLACKBOARD (BB)教学平台、moolsnet 微信公众号、以及 Mlabs 移动端虚拟仿 真实验软件, 在大一年级开展无机化学基础实验的线上教学课程。本文以氧化还原反应实验为例, 详细介绍具体的教学设计策略和教学实施过程, 在提供给大家一个 “完全线上” 的远程实验教学案 例的同时, 以问题导向来增强教学互动, 通过设计多环节教学过程和实行多方位考核评价来提升教 学效果, 从而将实验课程的线上教学做实做好。

\section{2 教学设计}

图 1 给出了实验教学线上课堂的整体构建框架。

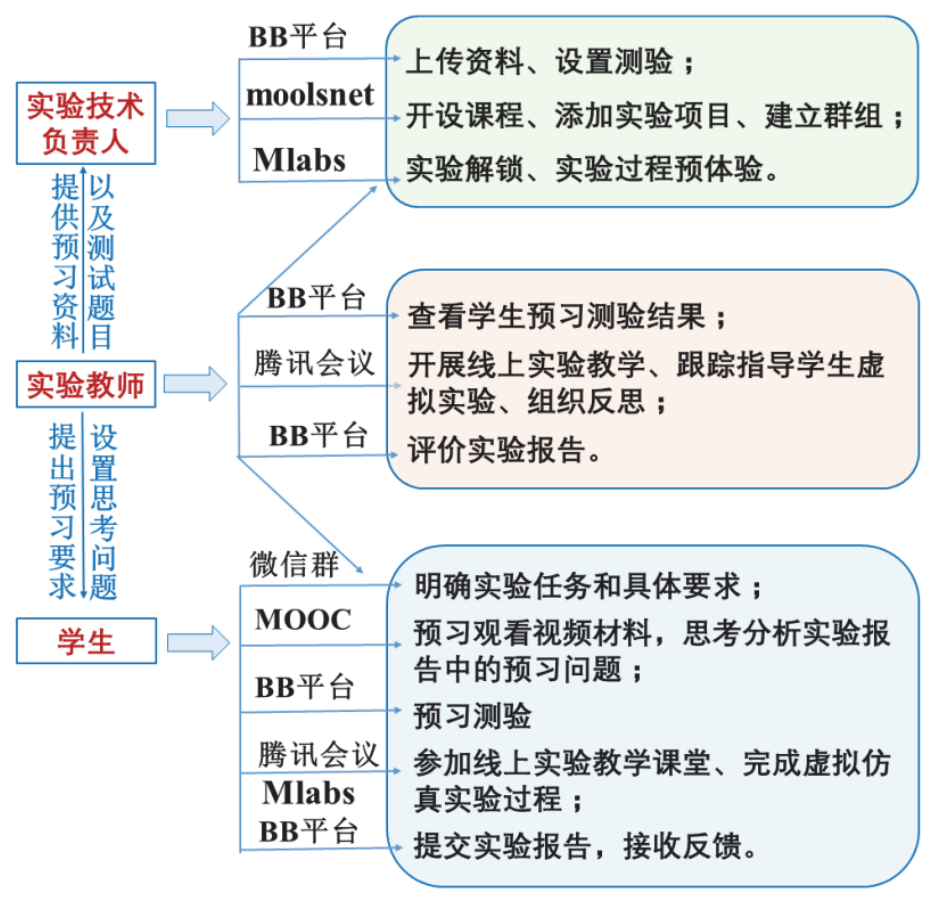

图 1 实验教学线上课程的整体构建框架

按照教学规划, 本学期初学生刚学完氧化还原反应理论部分, 同步开展氧化还原反应的线上实 验教学有助于巩固理论知识, 加强理论知识与实验内容之间的关联性, 并提高学生运用理论知识分 析解释实验现象的能力。

巩固电极电势与氧化还原反应的关系; 加强理解介质的酸碱性对氧化还原反应方向和产物的影 响; 加强理解反应物浓度和温度对氧化还原反应速率的影响; 掌握浓度对电极电势的影响; 巩固固 液取用、混合反应、加热、点滴板使用等基本操作, 学习测定原电池电动势的方法; 通过问题讨论 和课后实验设计训练学生的科学思维, 培养学生的科研能力和综合运用知识的能力。 
实验采用 20 人以内小组教学, 灵活运用腾讯会议(屏幕共享、语音交流、对话框讨论区)开展线 上问题启发式和讨论交流的互动式教学过程, 引导学生逐步进行自主预习测验、分析思考、设想实 验情境、虚拟仿真验证、讨论实验结果、总结反思与拓展的学习过程。

师生共同进行中国大学 MOOC 注册、moolsnet 微信公众号实名注册与认证, 手机端下载 Mlabs 软件; 实验负责教师上传预习资料到 BB 平台, 设置预习测验, 同时在 moolsnet 系统建立实验课程、 项目内容和学生群组信息, 便于收集整理 Mlabs 虚拟仿真实验的成绩结果与实验操作过程的反馈信 息。教师主要使用电脑, 配合使用手机; 学生使用手机即可, 电脑可用于辅助完成电子版实验报告。

\section{3 教学实施}

根据实验课的特点和教学目标要求, 综合各种教学资源, 设计了 “线上课前准备、线上互动教 学、线上反思总结与课后拓展”实验教学过程。具体的教学实施开展如下。

\section{1 线上课前准备与评价}

课前准备包括 MOOC 视频学习、预习思考分析以及预习测验三个部分。图 2 给出了本实验的实 验报告模板和 MOOC 中的实验操作演示截图。

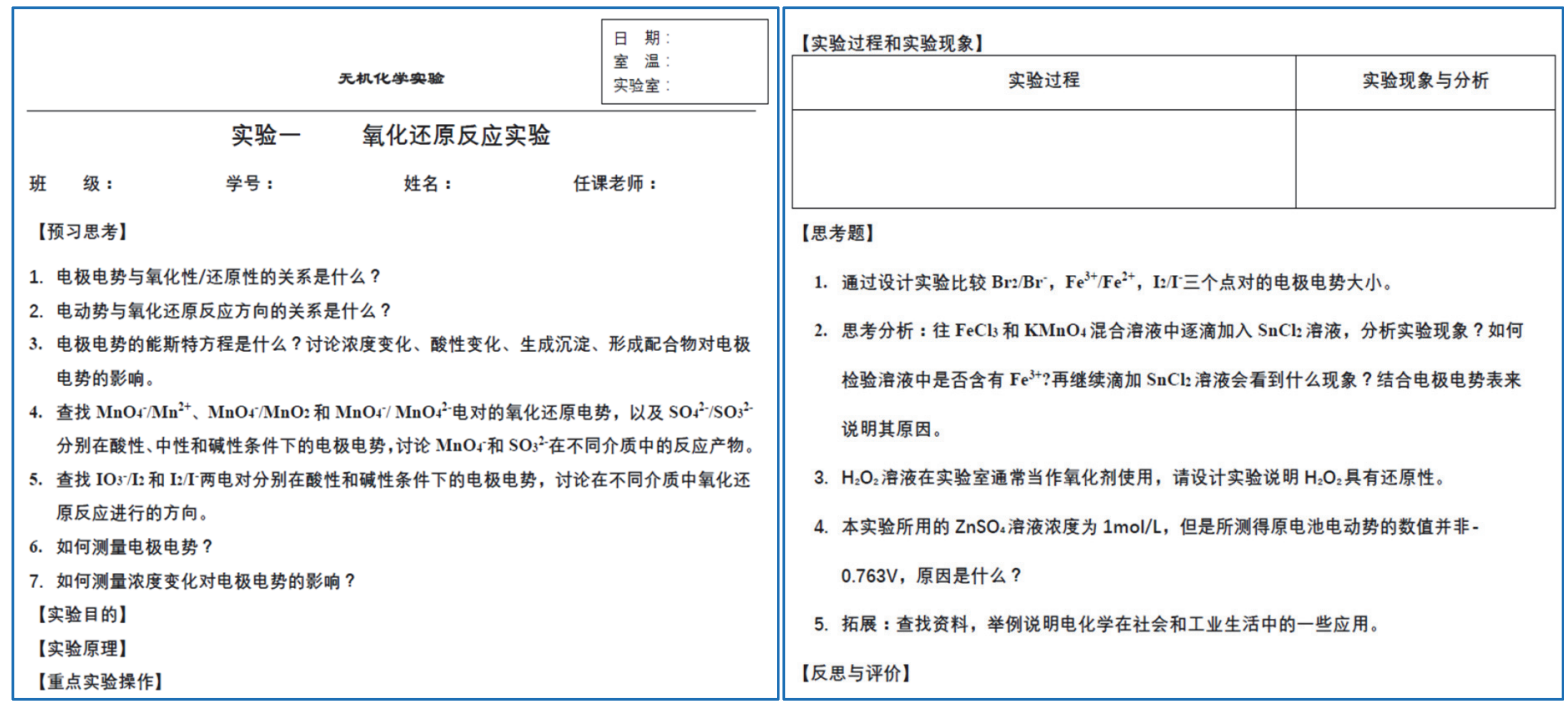

实验报告模板

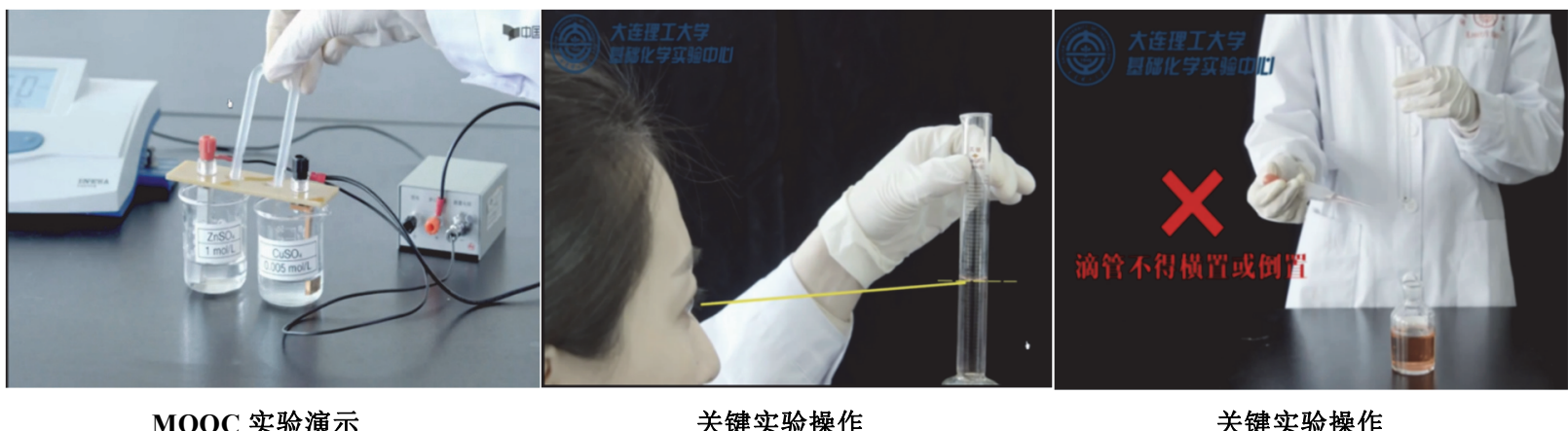

图 2 本实验的实验报告模板和 MOOC 中的实验操作演示截图

基于 MOOC 资源的支撑, BB 平台中仅上传了实验报告模板和实验内容讲义。借助 MOOC 中 的视频资源, 学生对实验内容和实验原理进行初步了解, 并详细学习实验中的关键操作, 同时通过 
观看演示实验短片熟悉实验过程。在此基础上，对实验报告中的预习思考题进行分析和充分准备。 在实验课程教学之前, 学生需要登录 $\mathrm{BB}$ 平台完成实验测验。图 3 为本次实验中学生的预习测验成 绩概况。根据 BB 平台的成绩反馈, 大一年级 286 位学生共尝试测验 324 次, 平均成绩为 88.48 分。 图 3 中的问题难度表现为正确率的实际测试情况, 从中可以看出, 难度在 $90 \%$ 以上和以下的题型各 占 $50 \%$ 。平均成绩在 85 分以上, 说明学生对于氧化还原反应知识的总体掌握情况良好, 但仍存在个 别知识盲点: 比如盐桥的作用以及酸度对电极反应的影响情况。预习测验的设置有助于学生检验预 习工作和对知识的掌握情况, 进而激发学生学习的主动性, 同时可以作为课堂补充内容以供教师进 行讲解和强调。

\begin{tabular}{|c|c|c|c|c|}
\hline & 问题 & 问题类型 & 对比 $\nabla$ & 难度 \\
\hline • & 下列电对的电极电势受溶液酸度影响的是（） & 单项选择题 (RB) & 0.71 & $81.25 \%$ \\
\hline • & $\mathrm{Cl} / 2 / \mathrm{Cl}-\mathrm{Hg} 2+/ \mathrm{Hg} ， \mathrm{Fe} 3+/ \mathrm{Fe} ， \mathrm{Sn} 2+/ \mathrm{Sn}$ 四个电对的标准电极电势分别为 $1.36 \mathrm{~V} 、 0.85 \mathrm{~V} 、 0.77 \mathrm{~V}$ 和 $-0.14 \mathrm{~V}$ ，下列不可共存于... & 单项选择题 (RB) & 0.64 & $83.04 \%$ \\
\hline • & 盐桥中的电解质不参与电极反应？（ ） & 单项选择题 (RB) & 0.61 & $96.43 \%$ \\
\hline & 对于Cu2+/Cu电极，下列说法正确的是？ & 单项选择题 (RB) & 0.56 & $72.33 \%$ \\
\hline • & $\mathrm{KClO} 3 、 \mathrm{Br} 2 、 \mathrm{FeCl} 、 \mathrm{KMnO} 4$ ，当溶液中 $\mathrm{H}+$ 浓度增大时，氧化能力增强的是 （\&nb... & 单项选择题 (RB) & 0.56 & $87.5 \%$ \\
\hline • & 下列说法正确的是 ( ) & 单项选择题 (RB) & 0.55 & $80.36 \%$ \\
\hline • & 浓度对氧化还原学反应速率的影响是（） & 单项选择题 (RB) & 0.49 & $95.54 \%$ \\
\hline$\bullet$ & 物质氧化还原能力与电极电势的关系是（） & 单项选择题 (RB) & 0.43 & $96.43 \%$ \\
\hline$\bullet$ & 将H2O2加入硫酸酸化的KMnO4湻液中， $\mathrm{H} 2 \mathrm{O} 2($ （） & 单项选择题 (RB) & 0.42 & $91.97 \%$ \\
\hline - & 饱和甘录电极与标准甘录电极的电极电势相等？ & 单项选择题 (RB) & 0.28 & $96.43 \%$ \\
\hline
\end{tabular}

图 3 BB 平台中学生的预习测验成绩概况

\section{2 线上互动教学}

为了增强教学互动并体现学生的课堂主体性, 我们根据学生的参与情况将实验课堂细分为前期 问题导入与解决、中期实验操作体验、后期实验操作分析 3 个密切相关的过程。

(1) 前期过程采用问题导向与讨论交流的模式展开, 由教师引导, 以问题为基础, 通过师生互动 或生生互动来解决问题, 有利于锻炼学生的思辨能力和创新能力, 激发学生的科研热情并提升创新 意识。线上教学的内容包含巩固预习测验、提出实验目的、讨论实验原理、分析实验过程、讲解关 键操作、强调实验注意事项等方面。在氧化还原反应的线上教学过程中, 为了增强互动, 提升教学 效果, 我们将预习思考中的问题分别以教师提问(语音)、自主发言(语音), 以及讨论方式逐步落实并 解决。由于事先已经进行充分有效的预习, 学生在线上教学过程的参与情况表现积极, 而且回答问 题的正确率很高。更为重要的是, 根据反馈信息, 学生对这种通过事先思考、设置课堂提问和讨论 交流从而获取知识并提升问题分析与解决能力的效果非常满意。图 4 为在腾讯会议开展氧化反应实 验线上教学中的课堂提问与讨论交流活动展示图片。

(2) 中期过程主要运用 Mlabs 虚拟仿真实验平台完成线上实验操作体验。学生通过微信登录虚 拟仿真实验平台, 进入氧化还原实验页面, 逐步完成 $\mathrm{KMnO}_{4}$ 与 $\mathrm{Na}_{2} \mathrm{SO}_{3}$ 溶液在酸性、中性以及碱性 介质中的反应实验、 $\mathrm{KIO}_{3}$ 与 $\mathrm{KI}$ 依次加入稀 $\mathrm{H}_{2} \mathrm{SO}_{4}$ 和 $\mathrm{NaOH}$ 溶液时的反应实验、溶液中 $\mathrm{Pb}^{2+}$ 浓度对 $\mathrm{Zn}$ 置换 $\mathrm{Pb}$ 反应速率的影响实验、反应温度对 $\mathrm{KMnO}_{4}$ 与草酸溶液反应速率的影响实验、标准 $\mathrm{Zn}$ 电 极的电极电势测定实验, 以及形成配合物所导致的浓度变化对 $\mathrm{Cu}$ 电极的电极电势的影响实验。Mlabs 软件在实验考核模式下依然存在实验操作步骤信息提示。为了切实锻炼提高学生的实验能力, 我们 要求学生在关闭信息提示的情况下开展实验。而且建议学生在进行实验考核之前, 根据前期课堂教 


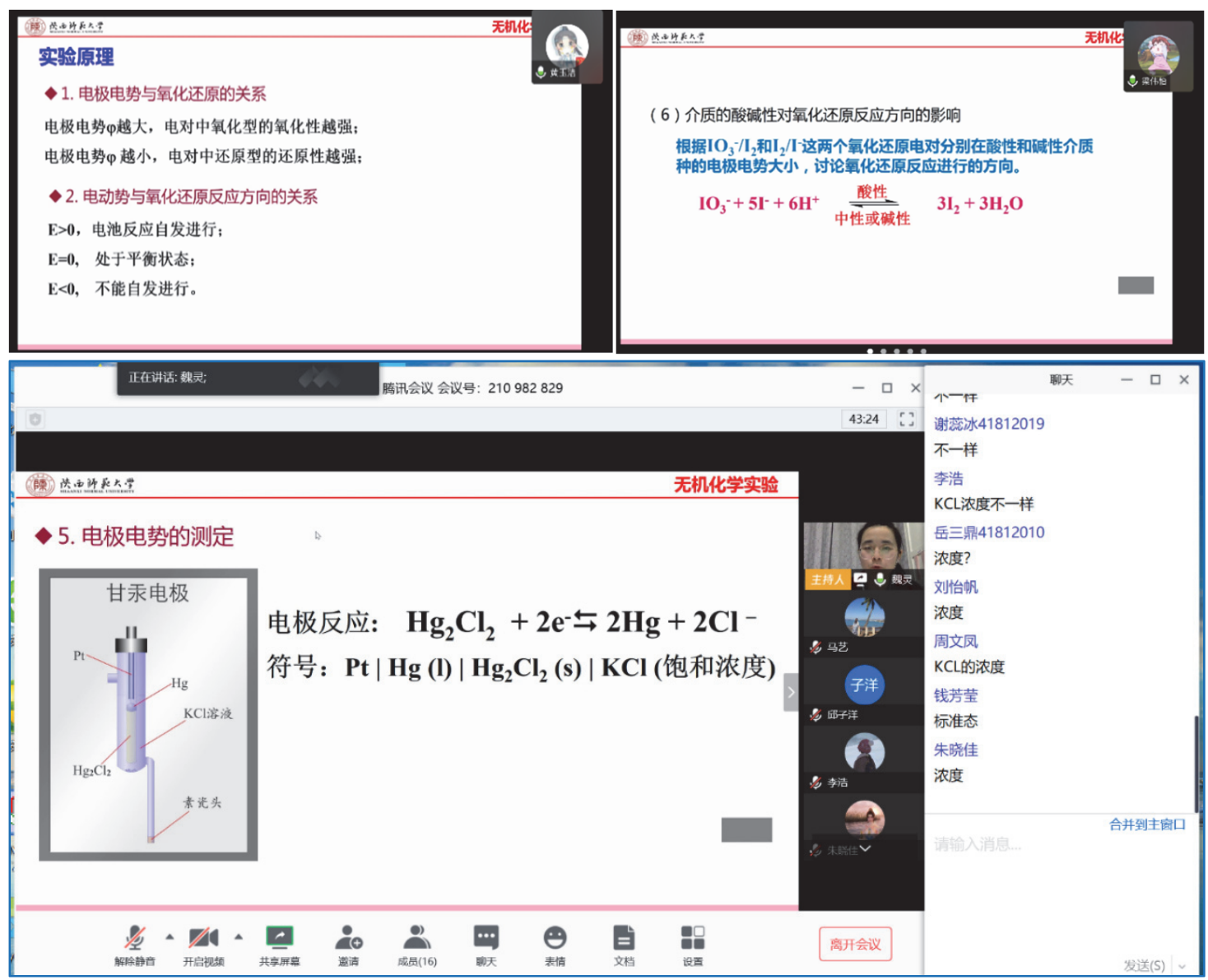

图 4 腾讯会议开展氧化反应实验线上教学中的课堂提问与讨论交流活动展示

学内容预设实验过程画面, 并在实验考核中进行对比和实践, 通过还原线下实验操作的真实场景以 加强学生的切身感受。

图 5 给出了学生在 Mlabs 手机端进行线上虚拟仿真实验的操作图片。借助腾讯会议中的屏幕分 享模式, 学生可以将具体实验过程的画面分享展示给老师与其他同学, 以实现相互交流与协作。由 于大一学生在上学期基础化学实验教学中已经学习并熟练掌握固液取用, 试管、胶头滴管、量筒、 $\mathrm{pH}$ 试纸的使用, 液体加热这些基本操作, 本虚拟实验过程对于学生来说除了学习电极电势的测定方 法之外，主要侧重于体会实验内容设计、强化理论与现象的关联性、提升知识运用能力。

(3) 后期过程主要实施实验操作的分析探讨。实验完成之后, moolsnet 教师系统可以刷新出学生 的线上实验成绩。针对实验教学内容和虚拟实验操作过程的重要问题, 通过腾讯会议平台进行巩固 与强化。在此部分我们设置的问题是: 在 $\mathrm{Zn}$ 置换 $\mathrm{Pb}$ 的实验中, 为什么要加入 $\mathrm{HAc}$ 和 $\mathrm{Na}_{2} \mathrm{SiO}_{3}$ 溶 液? 能不能加入硫酸或者盐酸人来代替 HAc? 在测量标准 $\mathrm{Zn}$ 电极电势的实验中, 为什么获得的实 验结果并不是 $-0.763 \mathrm{~V}$ ? 比较欣喜的是, 根据 moolsnet 成绩反馈, 在本实验的课堂设计和实施模式 下, 高达 $99 \%$ 的学生在没有任何信息提示的情况下都能达到 100 分的实验成绩, 说明切实实现了教 学目标中针对学生知识运用和能力提升的要求。

\section{3 线上反思总结与课后拓展}

我们采用腾讯会议中开启语音和讨论区模式进行讨论总结。首先引导学生通过思考、讨论交流 与展示汇报完成两个实验设计过程, 分别为: 设计实验比较 $\mathrm{Br}_{2} / \mathrm{Br}^{-}, \mathrm{Fe}^{3+} / \mathrm{Fe}^{2+}, \mathrm{I}_{2} / \mathrm{I}^{-}$三个电对的电极 
电势大小; $\mathrm{H}_{2} \mathrm{O}_{2}$ 溶液在实验室通常当作氧化剂使用, 请设计实验说明 $\mathrm{H}_{2} \mathrm{O}_{2}$ 具有还原性。图 6 为实 验设计案例展示, 通过实验过程的设计, 可明显提升学生的思辩能力和灵活运用理论知识的能力, 并进一步将学生在虚拟仿真实验中体验过的实验过程和细节步骤进行应用。
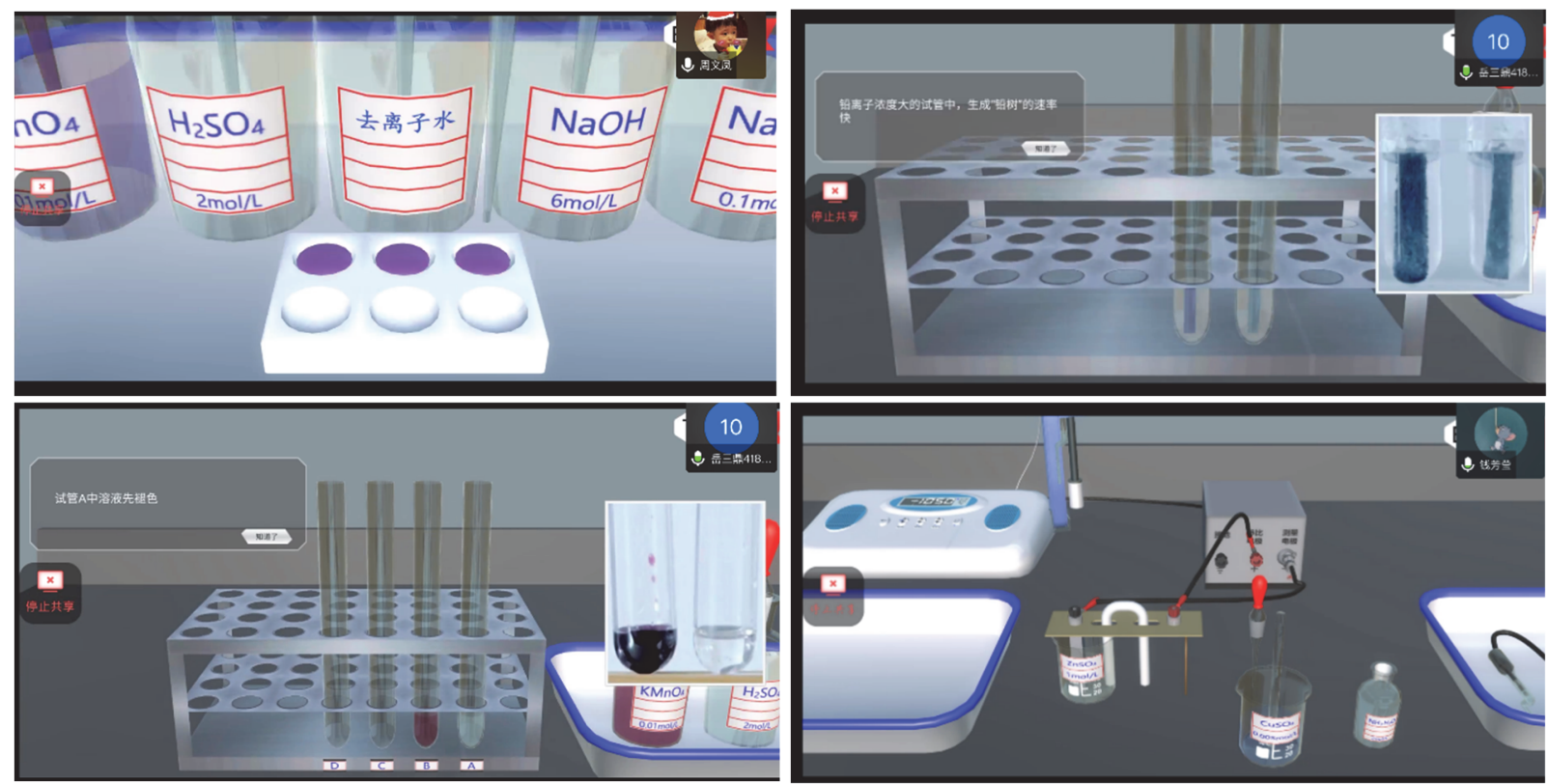

图 5 学生在 Mlabs 手机端进行线上虚拟仿真实验的操作图片

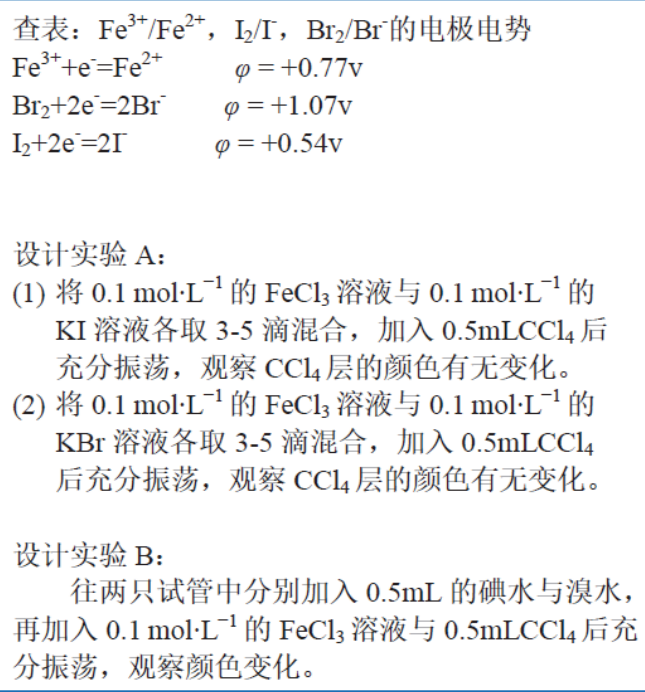

(1) 将 $0.1 \mathrm{~mol} \cdot \mathrm{L}^{-1}$ 的 $\mathrm{FeCl}_{3}$ 溶液与 $0.1 \mathrm{~mol} \cdot \mathrm{L}^{-1}$ 的 $\mathrm{KI}$ 溶液各取 3-5 滴混合, 加入 $0.5 \mathrm{mLCCl}_{4}$ 后 充分振荡, 观察 $\mathrm{CCl}_{4}$ 层的颜色有无变化。

(2) 将 $0.1 \mathrm{~mol} \cdot \mathrm{L}^{-1}$ 的 $\mathrm{FeCl}_{3}$ 溶液与 $0.1 \mathrm{~mol} \cdot \mathrm{L}^{-1}$ 的 $\mathrm{KBr}$ 溶液各取 3-5 滴混合, 加入 $0.5 \mathrm{mLCCl}_{4}$ 后充分振荡, 观察 $\mathrm{CCl}_{4}$ 层的颜色有无变化。

\section{设计实验 $\mathrm{B}$ :}

往两只试管中分别加入 $0.5 \mathrm{~mL}$ 的碘水与溴水, 再加入 $0.1 \mathrm{~mol} \cdot \mathrm{L}^{-1}$ 的 $\mathrm{FeCl}_{3}$ 溶液与 $0.5 \mathrm{mLCCl}_{4}$ 后充 分振荡, 观察颜色变化。

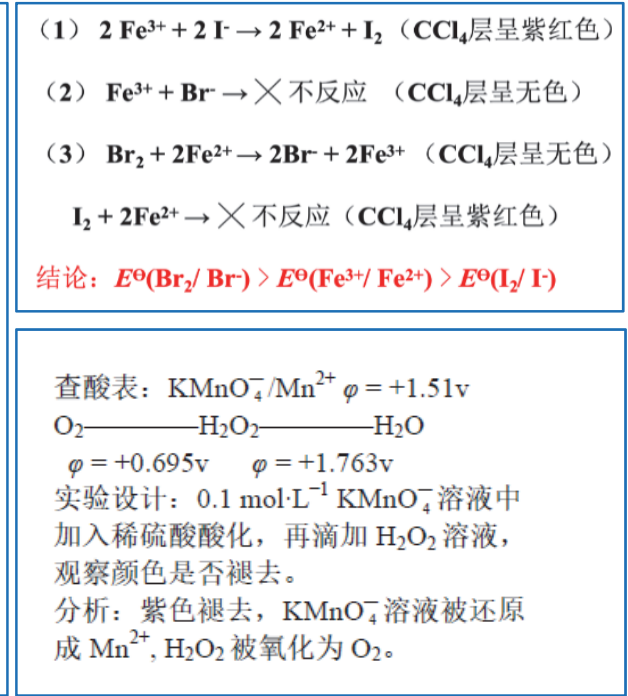

图 6 学生参与实验设计案例展示

随后, 学生线上完成实验报告, 并提交到 BB 教学平台的作业区域, 教师进入 BB 教学平台实 时进行教学评价与反馈。由于预习思考、实验目的和实验原理已在线上课堂进行讲解, 实验报告的 成绩主要参考实验过程与现象分析、思考问题, 以及反思与评价部分来评定。另外, 在具体实验教 学过程中, 我们也一直反思总结在促进学生理论联系实验以及锻炼学生实验能力的同时如何进一步 提升学生的创新思维和科学素养。氧化还原性实验从总体来说, 实验操作简单, 知识的理论性较强, 因此, 我们要求学生将思考问题中的电化学在社会生活中的应用作为开放性课题, 通过查阅和整合 
资料, 3-4 人为一组, 撰写一篇简要论述, 具体内容可从以下课题中选择: 电池的发展史、锂离子电 池的原理与应用、锂离子电池电极材料的研究进展、电解池在工业生产中的应用、电化学在水处理 中的应用、电解水制氢的研究等。

\section{4 线上实验教学考核与展望}

针对氧化还原线上实验教学, 为了对学生实验情况作出客观合理的评价, 实验总成绩由 5 部分 组成: 其中, 预习测验占 $30 \%$ 的比例, 实验过程分数占 $20 \%$ 的比例, 实验报告占 $30 \%$ 的比例, 知识 拓展占 $10 \%$ 的比例, 还有 $10 \%$ 的成绩比例用于体现学生参与课堂教学过程的问题讨论、互动和互评 行为。

在以上评价体系下, 绝大多数同学实验总评成绩在 80 分以上, 只有极少数同学成绩在 80 分以 下。说明本实验中测验题目的设置、预习与思考问题的设计, 以及总评成绩分配情况是合理可行的。 但也存在一些问题需要重视和解决：思考题回答有误、针对线上实验不知道如何进行反思与评价; 在知识拓展方面, 有绝大一部分学生缺乏主动探索的兴趣和意识, 电化学的应用论述过于陈旧, 缺 乏科学研究动态与技术前沿介绍。

此外, 为了贯彻落实以学生为中心的指导思想和以能力为先的人才培养理念, 从而使线上课程 教学在后续工作中能够得到进一步改进和完善, 我们还特别注重引导学生总结实验收获, 展示自己 的问题, 并提出意见或建议。针对大一学生, 在疫情期间我们已经开展了 3 次线上科研类虚拟仿真 实验与操作体验和 2 次线上基础类虚拟仿真实验与操作体验。图 7 给出了本校大一学生对于开展两 种类型的虚拟仿真实验线上教学的评价、调查反馈以及本次实验成绩分布情况。从中可以看出, 尽 管我们对线上实验教学进行了详细设计, 学生的实验效果和成绩还比较满意。但大一学生由于理论 知识不够完善、实验技能不够健全、科学前沿了解较少, 以及缺乏现代实验表征技术基础, 在我们 的调查取样中有将近 70\%的同学还是最期望基础实验的线下操作模式, 有 $27 \%$ 的同学对基础实验的 线上实验操作模式表现出强烈的兴趣，仅有 $3 \%$ 的同学对科研类实验的线上虚拟仿真实验比较期待。

\begin{tabular}{ll}
\hline 4 & 无机化学(42) \\
更喜欢基础类虚拟仿真 \\
实验体验: 基础虚拟仿 \\
真每步实验后, 会出现 \\
实验现象, 比科研类的 \\
更有真实感。 \\
知识收获: 基础的更适 \\
合一些, 因为目前我的 \\
知识体系还不够完善, \\
基础类的现象能够自己 \\
思考与理解, 而科研类 \\
的做完实验就忘完了, \\
能理解的很少。 \\
能角度: 基础实验目前 \\
已经接触了一学期, 已 \\
经有了一些经验。科研 \\
类的有些仪器, 没有接 \\
触过, 也不太了解原 \\
理, 而且网上操作没有 \\
实际操作更好。
\end{tabular}

(ग))

\begin{tabular}{cc}
$\cdots<21$ & \multicolumn{1}{c}{ 无机化学(42) } \\
\hline 我更喜欢基础类虚拟仿 \\
真实验, 基础类虚拟仿 \\
真实验在知识获取上更 \\
为充分, 较之科研类更 \\
为充分掌握其机理和相 \\
关实验操作。在巩固完 \\
基础知识后, 会以更大 \\
的视角进行实验, 实验 \\
起来更加得心应手, 更 \\
充分的将理论与实践充 \\
分结合, 实验过后所获 \\
的知识也更加牢固。但 \\
科研类也有其好处, 在 \\
历尽千辛万苦查阅资料 \\
的过程中会充满趣味 \\
性, 更加使你了解前瞻 \\
性的仪器, 设备, 反应 \\
机理等, 会拓宽你的视 \\
野。
\end{tabular}
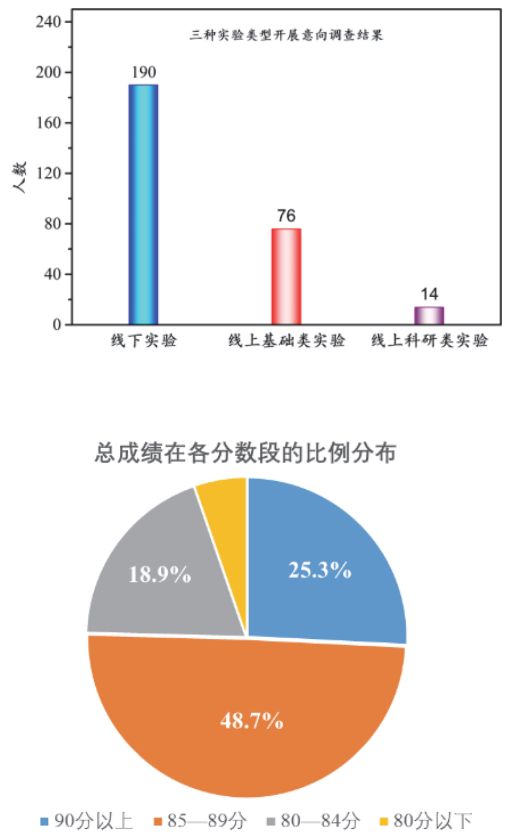

图 7 大一学生对于开展虚拟仿真实验线上教学的评价(左)、调查反馈(右上)以及本次实验成绩分布情况图(右下) 
与线下实验相比, 线上实验在丰富教学内容和改变教学方式上更为灵活多样, 但学生对于实验 操作细节和实验过程的印象会淡化很多。因此, 在学生返校之后, 我们计划先开展一次制备合成实 验以巩固练习基本操作技能, 并以实验考核的方式从近期开展的线上实验教学内容中选取重要素材 补做一次线下实验, 在强化线上实验教学效果的同时做好线上与线下实验教学的衔接。此外, 在后 续的实验教学改革中, 我们继续保持传统线下实验操作的主体地位, 同时根据授课对象和实验内容 特点, 选择性引入一些基础类虚拟仿真实验和科研类虚拟仿真实验, 篎选、整合、加工各种教学资 源, 全方位服务于学生, 既能加强学生综合能力和创新能力的培养, 也可以提升教师的教学技能, 并发展和完善无机化学实验课程建设。

\section{5 结语}

基于中国大学 MOOC 平台课程资源、依托 BLACKBOARD 教学平台、以微信群作为交流媒介、 借助腾讯会议和 Mlabs 虚拟仿真软件, 我们构建了多元化和立体化的氧化还原实验线上实验课堂。 通过问题导向将实验预习、教学互动、实验操作体验、知识总结、反思评价高效串联, 切实实现了 预定的教学目标。通过实验教学探索和实施, 我们体会到线上教学不是线下教学的照搬, 更不是线 下教学的对亦, 实验课程的选择和设计是实施线上实验教学的核心。因此, 针对实验教学, 如何借 助线上课堂的优势, 将线上与线下有效结合, 虚实互补虚实共促, 从而在实验教学中贯彻落实 “以 学生为中心” 的指导思想和以能力为先的人才培养理念, 进一步提升学生的创新意识和科研素养, 使得实验教学在为国家培养创新应用型人才的过程中起到应有的作用, 还需要我们一如既往地追求 和探索。

\section{参 考 文 献}

[1] 王兴伟, 李婕, 谭振华, 马连博, 李福亮, 黄敏. 计算机研究与进展, 2016, 53 (4), 729.

[2] 曾明星, 李桂平, 周清平, 覃遵跃, 徐洪智, 张彬连, 黄云, 郭金鍂. 中国电化学教育, 2015, 36 (11), 28.

[3] 谢爱娟, 罗士平, 孔泳，马江权. 实验室技术与管理, 2017, 34 (9), 211.

[4] 王秀云, 宿艳, 张永策, 潘玉珍. 大学化学, 2020, in press. doi: 10.3866/PKU.DXHX202001032

[5] 叶红, 何苏萍, 陈云, 俞松林. 化学教育, 2018, 39 (22), 37.

[6] 宿艳, 张永策, 杨金辉, 孟长功. 实验室科学, 2019, 22 (4), 120.

[7] 谢爱娟, 陶宇炜, 罗士平, 薛冰. 化学教育, 2018, 39 (14), 44

[8] 杜静, 马骁飞, 赵温涛. 大学化学, 2020, in press. doi: 10.3866/PKU.DXHX201912048

[9] 张玮玮, 弓爱君, 邱丽娜. 大学化学, 2020, 35 (3), 94.

[10] 胡菁, 周金梅, 陈立. 大学化学, 2020, in press. doi: 10.3866/PKU.DXHX202001005

[11] 张瑶. 大学化学, 2020, 35 (3), 57.

[12] 龚成斌, 曹雅仪, 王强, 唐倩, 张浩. 西南师范大学学报, 2019, 44 (11), 155. 